\title{
A DESCRIPTION OF STOCHASTIC SYSTEMS USING CHAOTIC MAPS
}

\author{
ABRAHAM BOYARSKY AND PAWEŁ GÓRA
}

Received 28 August 2003 and in revised form 4 February 2004

Let $\rho(x, t)$ denote a family of probability density functions parameterized by time $t$. We show the existence of a family $\left\{\tau_{t}: t>0\right\}$ of deterministic nonlinear (chaotic) point transformations whose invariant probability density functions are precisely $\rho(x, t)$. In particular, we are interested in the densities that arise from the diffusions. We derive a partial differential equation whose solution yields the family of chaotic maps whose density functions are precisely those of the diffusion.

\section{Introduction}

In this paper, we establish a method for describing flows of probability density functions by means of discrete-time chaotic maps. We start with a standard map whose invariant probability density function is known and then use it to derive other invariant probability density functions by a simple conjugation process which solves the inverse PerronFrobenius problem $[2,3]$ in a time-varying setting.

\section{Notation and preliminary results}

In this paper, we consider space to consist of 1 dimension although the extension to 2 and 3 dimensions is straightforward. In the sequel, we will need some notions from ergodic theory and nonlinear dynamics, which can be found in [1].

Let $\mathbb{R}=(-\infty, \infty)$ and let $T: \mathbb{R} \rightarrow \mathbb{R}$ possess a unique absolutely continuous invariant measure $\mu$ which has the probability density function $f$, that is,

$$
\int_{A} f d x=\int_{T^{-1} A} f d x
$$

for any measurable set $A \subset \mathbb{R}$. The Perron-Frobenius operator $P_{T}$ acting on the space of integrable functions is defined by

$$
\int_{A} f d x=\int_{T^{-1} A} P_{T} f d x
$$


The operator $P_{T}$ transforms probability density functions into probability density functions under the transformation $T$, where $T$ is assumed to be nonsingular. One of the most important properties of $P_{T}$ is that its fixed points are the densities of measures invariant under $T[1]$.

Let $h: \mathbb{R} \rightarrow \mathbb{R}$ be a diffeomorphism. Then $\tau=h^{-1} \circ T \circ h$ is a transformation from $\mathbb{R}$ into $\mathbb{R}$, which is differentiably conjugate to $T$ and whose probability density function is given by

$$
k=(f \circ h) \cdot\left|h^{\prime}\right|
$$

We assume that $T$ is a piecewise monotonic expanding $C^{1}$ map on $\mathbb{R}$ that admits a unique absolutely continuous invariant measure. Then the invariant density function $f(x)$ is a fixed point of the Perron-Frobenius operator $P_{T}[1]$. We now consider the inverse Perron-Frobenius problem: suppose we are given a probability density function $g(x)$ on $\mathbb{R}$, can we find a transformation $\tau$ such that $g(x)$ is the unique probability density function invariant under $\tau$ ? This problem has been dealt with by Ershov and Malinetskiu [2] and in [3] from a computational perspective.

We solve the inverse Perron-Frobenius problem by applying (2.3), that is, we find $h$ such that

$$
(f \circ h) \cdot h^{\prime}=g
$$

where we have assumed, without loss of generality, that $h$ is an increasing function on $\mathbb{R}$. Now, let

$$
F(x)=\int_{-\infty}^{x} f(y) d y
$$

be the distribution function associated with $f$. Then, from (2.4) and the change-ofvariable formula, we have

$$
F(h(x))=\int_{-\infty}^{x} g(y) d y .
$$

Since $F$ is a monotonically increasing function, it has a unique inverse and

$$
h(x)=F^{-1}\left(\int_{-\infty}^{x} g(y) d y\right) .
$$

Thus, we have found $h(x)$ such that $\tau=h^{-1} \circ T \circ h$ has the probability density function $g(x)$. Summarizing, given any probability density function $g(x)$, we have proven the existence of a point transformation $\tau$ whose probability density function is $g(x)$.

Example 2.1. Let

$$
T(x)=a \tan x, \quad x \neq \frac{k \pi}{2}, k= \pm 1, \pm 3, \ldots,
$$


and $a>1$. Then the probability density function invariant under $T$ is [1]

$$
f(x)=\frac{p}{\pi\left(p^{2}+x^{2}\right)}
$$

where $p>0$ satisfies the equation $a \tanh (p)=p$. For $p>2, a \approx p$ and we can assume that $T(x)=p \tan x$. Hence, for $p=4$, say,

$$
\begin{aligned}
f(x) & =\frac{4}{\pi\left(16+x^{2}\right)}, \\
F(x) & =\int_{-\infty}^{x} \frac{4}{\pi\left(16+y^{2}\right)} d y=\frac{1}{2}+\frac{1}{\pi} \arctan \left(\frac{x}{4}\right), \\
F^{-1}(x) & =-4 \cot (\pi x) .
\end{aligned}
$$

Now, suppose we want to find a map $\tau$ whose unique invariant probability density function is given by $g(x)=(\cos 8 x) \exp \left(-3 x^{2}\right)$. We obtain

$$
\int_{-\infty}^{x}(\cos 8 x) \exp \left(-3 x^{2}\right) d y=\frac{2 \exp \left(-2 x^{2}\right)(2 / \pi) \cos ^{2}(8 x)}{1+\exp (-64 / 3)}
$$

from which we can determine $h(x)$ using (2.7). Once $h$ is known, so is $\tau=h^{-1} \circ T \circ h$ whose probability density function is $g(x)$.

The foregoing method can be extended to a family of probability density functions $\left\{g_{t}(y): t \in I\right\}$. In this case, the homeomorphism $h$ becomes a family of homeomorphisms $\left\{h_{t}: t \in I\right\}$ parameterized by $t$, where

$$
h_{t}(x)=F^{-1}\left(\int_{-\infty}^{x} g_{t}(y) d y\right)
$$

\section{Chaotic map description of diffusions}

Consider the diffusion equation

$$
\frac{\partial \rho(x, t)}{\partial t}=-\nabla(v(x, t) \rho(x, t))=-\frac{\partial}{\partial x}\left[b(x, t) \rho(x, t)-\frac{1}{2} \frac{\partial \rho(x, t)}{\partial x}\right]
$$

where $b(x, t)$ is the forward drift coefficient. Our objective is to prove the existence of a family of point transformations $\left\{\tau_{t} \in \Gamma: t>0\right\}$ whose invariant probability density functions are $\left\{\rho_{t}: t>0\right\}$. To do this, we let $T$ be the transformation defined by (2.8) and we derive a partial differential equation for $h_{t}(x)$ such that $\left\{\tau_{t}=h_{t}^{-1} \circ T \circ h_{t}\right\}$ possesses $\{\rho(x, t): t>0\}$ as the associated family of invariant probability density functions.

Since

$$
h_{t}(x)=F^{-1}\left(\int_{-\infty}^{x} \rho(y, t) d y\right)
$$


we have

$$
\frac{\partial}{\partial t} h_{t}(x)=\left(F^{-1}\right)^{\prime}\left(\int_{-\infty}^{x} \rho(y, t) d y\right)\left(\int_{-\infty}^{x} \frac{\partial}{\partial t} \rho(y, t) d y\right) .
$$

Noting that

$$
\left(F^{-1}\right)^{\prime}\left(\int_{-\infty}^{x} \rho(y, t) d y\right)=\frac{1}{F^{\prime}\left(F^{-1}\left(\int_{-\infty}^{x} \rho(y, t) d y\right)\right)}
$$

and using (3.1), we obtain

$$
\frac{\partial}{\partial t} h_{t}(x)=\frac{1}{f\left(h_{t}\right)} \int_{-\infty}^{x}\left[-\frac{\partial}{\partial y} b(y, t) \rho(y, t)+\frac{1}{2} \frac{\partial^{2}}{\partial y^{2}}(\rho(y, t))\right] d y .
$$

Thus,

$$
f\left(h_{t}(x)\right) \frac{\partial}{\partial t} h_{t}(x)=-b(x, t) \rho(x, t)+\frac{1}{2} \frac{\partial}{\partial x}(\rho(x, t))+q(t)
$$

where $q(t)$ is an unknown function of $t$ only. To find $q(t)$, we return to (3.3) and write

$$
f\left(h_{t}(x)\right) \frac{\partial}{\partial t} h_{t}(x)=\frac{\partial}{\partial t} \int_{-\infty}^{x} \rho(y, t) d y
$$

We assume that $\rho(y, t)$ and $(\partial / \partial x)(\rho(x, t))$ both go to 0 as $x \rightarrow \infty$; then the right-hand side of (3.7) also goes to 0 since

$$
\int_{-\infty}^{\infty} \rho(y, t) d y=1
$$

for all $t \geq 0$. Hence, $q(t)=0$ for all $t \geq 0$. Thus, (3.6) reduces to

$$
f\left(h_{t}(x)\right) \frac{\partial}{\partial t} h_{t}(x)=-b(x, t) f\left(h_{t}(x)\right) \frac{\partial}{\partial t} h_{t}(x)+\frac{1}{2} \frac{\partial}{\partial x}\left(f\left(h_{t}(x)\right) \frac{\partial}{\partial t} h_{t}(x)\right)
$$

or

$$
(1+b(x, t)) f\left(h_{t}(x)\right) \frac{\partial}{\partial t} h_{t}(x)=\frac{1}{2} \frac{\partial}{\partial x}\left(f\left(h_{t}(x)\right) \frac{\partial}{\partial t} h_{t}(x)\right)
$$

whose solution is the family of homeomorphisms $\left\{h_{t}\right\}$ which determine the family of deterministic chaotic maps $\left\{\tau_{t}=h_{t}^{-1} \circ T \circ h_{t}\right\}$, whose probability density functions are equal to $\rho(x, t)$.

\section{Acknowledgment}

This research has been supported by Natural Sciences and Engineering Research Council of Canada (NSERC) grants. 


\section{References}

[1] A. Boyarsky and P. Góra, Laws of Chaos, Probability and Its Applications, Birkhäuser Boston, Massachusetts, 1997.

[2] S. V. Ershov and G. G. Malinetskiĭ, Solution of an inverse problem for the Perron-Frobenius equation, U.S.S.R. Comput. Math. and Math. Phys. 28 (1988), no. 5, 136-141 (Russian).

[3] P. Góra and A. Boyarsky, A matrix solution to the inverse Perron-Frobenius problem, Proc. Amer. Math. Soc. 118 (1993), no. 2, 409-414.

Abraham Boyarsky: Department of Mathematics and Statistics, Concordia University, 7141 Sherbrooke Street West, Montreal, Québec, Canada H4B 1R6

E-mail address: boyar@alcor.concordia.ca

Paweł Góra: Department of Mathematics and Statistics, Concordia University, 7141 Sherbrooke Street West, Montreal, Québec, Canada H4B 1R6

E-mail address: pgora@vax2.concordia.ca 


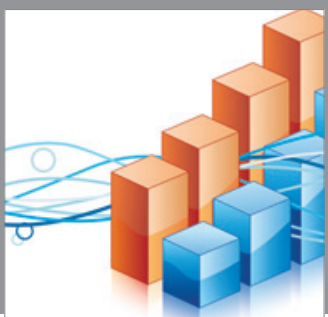

Advances in

Operations Research

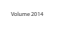

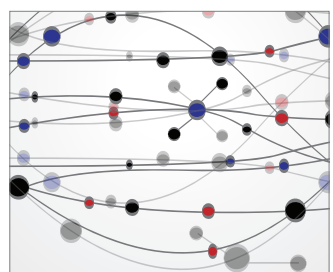

\section{The Scientific} World Journal
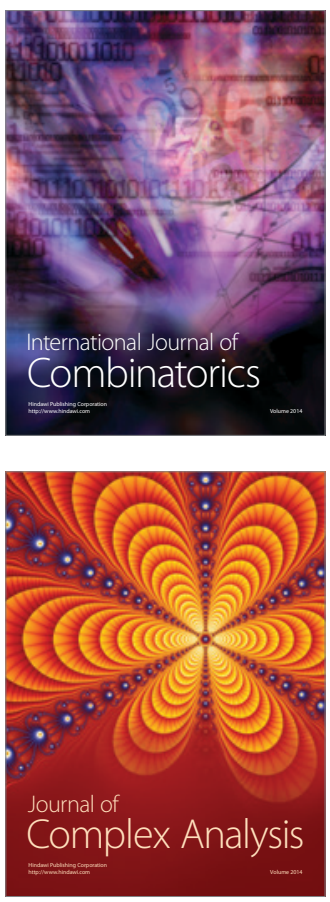

International Journal of

Mathematics and

Mathematical

Sciences
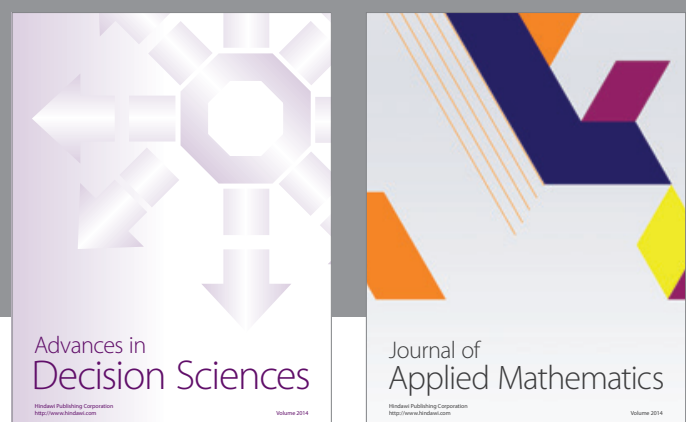

Journal of

Applied Mathematics
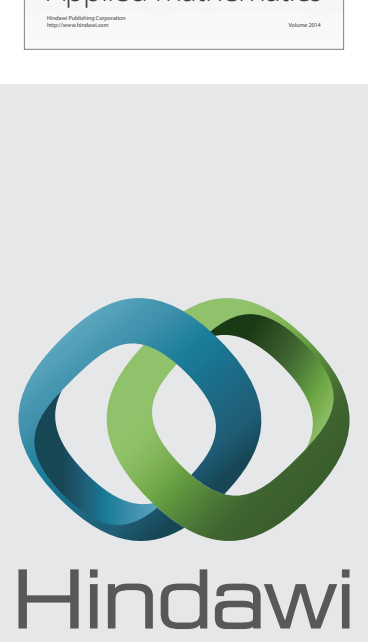

Submit your manuscripts at http://www.hindawi.com
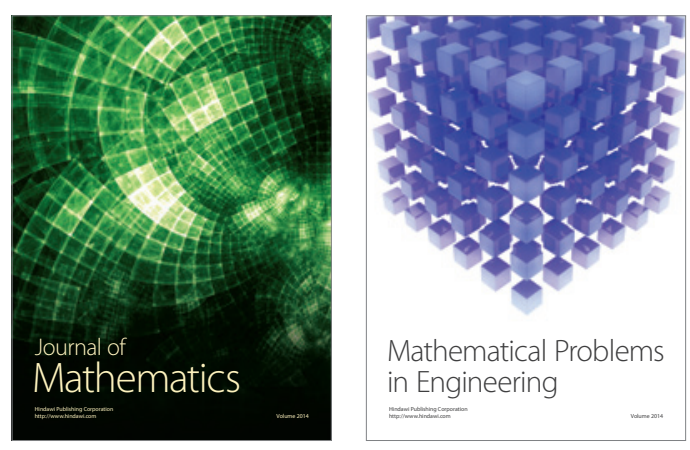

Mathematical Problems in Engineering
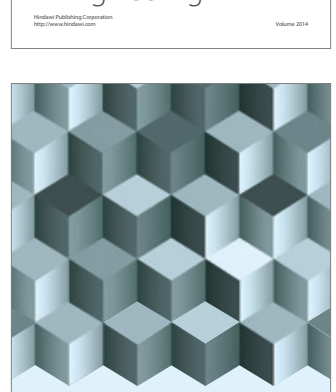

Journal of

Function Spaces
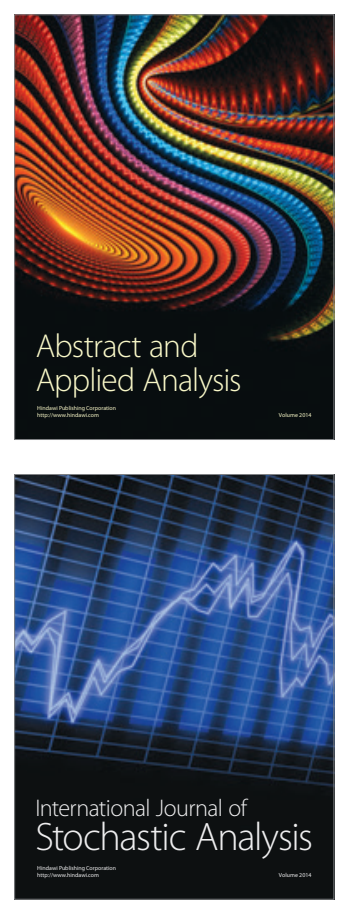

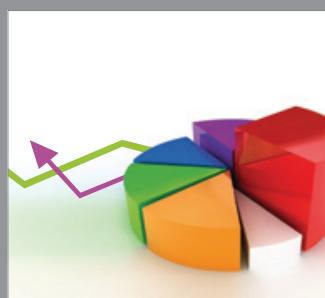

ournal of

Probability and Statistics

Promensencen
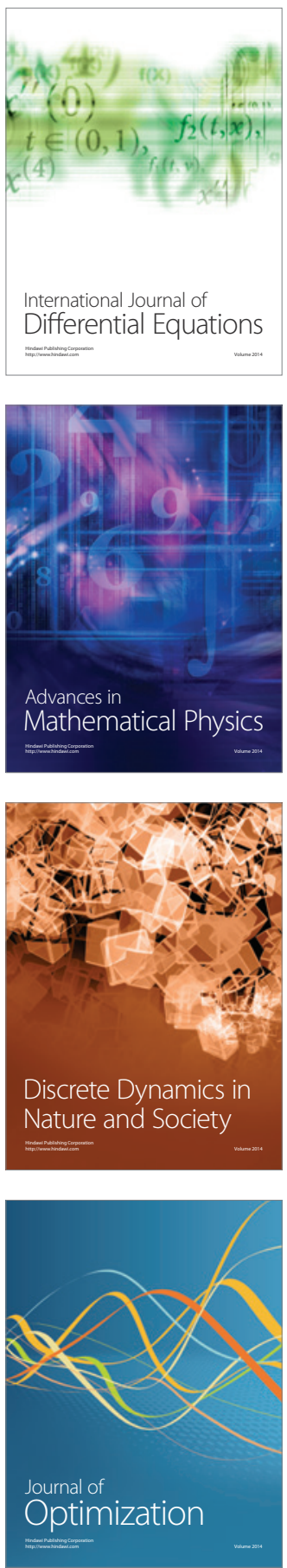